\title{
An evaluation of current perspectives on consciousness and pain in fishes
}

\author{
Kristopher Paul Chandroo, Stephanie Yue \& Richard David Moccia
}

Aquaculture Centre, Department of Animal and Poultry Sciences, University of Guelph, Guelph, ON, Canada N1G 2W1

\begin{abstract}
There is growing societal and scientific interest in the welfare status of fish used for commercial enterprise. As animal welfare is primarily concerned with the quality of life of a conscious, sentient organism, the question of whether fishes are even capable of consciousness must first be addressed in order to assess their welfare status. Recently, there has been a resurgence of research investigating the biological basis for human consciousness, and our current understanding of the cognitive mechanisms underlying fish behaviour has likewise improved significantly. Combined, these research perspectives create an opportunity to better comprehend the phylogeny of traits associated with consciousness, as well as the emergence of consciousness itself during vertebrate evolution. Despite the availability of this literature, contemporary reviews or published studies investigating the probability of conscious states occurring in fishes often do so without considering new perspectives or data. In this paper, we review and critique recent publications that report equivocal conclusions favouring the absence or presence of consciousness in various fishes. By introducing other data into these analyses, we demonstrate that there are alternative perspectives which support the existence of consciousness in fishes as a plausible concept. An accurate assessment of the mental capacity of fishes will require enhanced knowledge of their forebrain neuroanatomy, an understanding of how such structures mediate behavioural responses, and an analysis of that information within the context of contemporary theory related to the evolution of consciousness in higher vertebrates.
\end{abstract}

Keywords animal welfare, consciousness, fish, pain, sentience
Correspondence:

Richard David Moc-

cia, Aquaculture Cen-

tre, Department of

Animal and Poultry

Sciences, University of

Guelph, Guelph, ON,

Canada N1G 2W1

Tel.: (519) 824-4120

(ext. 56216)

Fax: (519) 767-0573

E-mail: rmoccia@

uoguelph.ca

Received 26 Jan 2004

Accepted 31 Aug 2004
Introduction

The neocortex and the neural correlates of consciousness

Convergence, homology and evolutionary psychology

286

Pain perception and brain structure

Cognition and behaviour in fishes

291

Conclusions

293

Acknowledgements

293

References

293 


\section{Introduction}

There is growing societal and scientific interest in the welfare status of fish used in commercial enterprise. As animal welfare is concerned with the quality of life of a conscious and sentient organism, the question of whether or not fishes are capable of conscious states must be addressed in order to evaluate their welfare status. Surprisingly, there is no universally accepted definition of consciousness as it applies across the spectrum of vertebrate phyla (Searle 2000). However, it is generally agreed among researchers that consciousness refers to a mental state of awareness of internal and external stimuli. Depending upon the quantitative or qualitative degree of awareness that is present in an organism, consciousness can also be described as existing in a primary or extended state (Lindahl 1997). Primary consciousness may be defined as the ability to generate a mental scene in which diverse information is integrated for the purpose of directing behaviour of self (Edelman and Tononi 2000). Extended consciousness is thought to involve 'higher order', advanced cognitive abilities that involve, for example, a linguistic capability or self-consciousness as self-knowledge (Zeman 2001). Regardless of whether an animal is thought to have primary or extended consciousness, both designations imply that they are sentient or self-aware organisms.

The probability of consciousness occurring in animals is typically assessed by comparing their neuroanatomical, behavioural and physiological characteristics with an array of human (or other well-studied mammalian) biological features that are closely associated with consciousness and emotional states. Comparative investigations of this kind have recently been published for fish species (Rose 2002; Sneddon 2003; Chandroo et al. 2004). The conclusions attained by simplistic, comparative assessment are often controversial, and much debate exists with respect to the accuracy of this analytical approach (Rolls 2000). This is especially true when such evaluations are focused on ancestral or 'primitive' vertebrate species, such as fish. Reasons for this controversy are wide ranging, and include philosophical disagreement concerning what comprises a legitimate form of scientific study (Searle 1998), as well as an historical bias regarding the interpretation of results derived from animal behaviour research (Griffin 1998; Schilhab 2002). Of equal controversy, is the disagreement with regard to the phylogenetic relationships between similar biological structures and their putative functions among distantly related species (Striedter 2002).

Until recently, the scientific investigation into the existence of conscious states in fishes has been compromised due to a lack of primary research investigating the aetiology of human consciousness, as well as a limited supply of comparative studies examining brain structure and cognition in fish species. However, a simultaneous resurgence of research investigating the neurophysiological basis of human consciousness, including telencephalic neuroanatomy and the underlying cognitive mechanisms of fish behaviour, has provided basic information that permits the phylogeny of biological traits associated with consciousness and consciousness itself to be studied more objectively (Butler and Hodos 1996; Baars 2002). Despite the availability of this information, current reviews or published studies investigating the probability of conscious states occurring in fishes, often do so without considering innovative, applicable data or alternative perspectives in its interpretation.

In this paper, we review and critique a number of recent publications that have reported equivocal conclusions on the existence of consciousness in fishes. We primarily critique the work of Rose (2002), and also address the work of Cabanac (1999), Sneddon (2003), Sneddon et al. (2003) and Cabanac and Cabanac (2000). Rose (2002) argues that it is most likely impossible for fish to experience pain or fear, while in contrast, Sneddon et al. (2003) provide anatomical, physiological and behavioural evidence that demonstrates nociception in fish, concluding that fish can also perceive pain. Based on empirical studies focused on physiological and behavioural responses, Cabanac (1999) suggests that fish do not have the ability for consciousness and emotion. The introductory list of issues and concepts found within (Rose 2002, p. 2) illustrate Rose's position that 'anthropomorphic thinking undermines our understanding of other species', and that 'an evolutionary perspective is essential to understanding the neurobehavioural differences between fishes and humans.' We agree with Rose that an unjustified ascribing of mental abilities to animals and the lack of an evolutionary perspective will lead to inaccurate conclusions with regard to the mental life of any animal. However, the evolutionary perspective that Rose presents is heavily dependent upon contrasts and anthropocentric 
arguments. Primary literature on the neurobiological features and learning behaviour of fishes seem lacking in Rose's review, despite the fact that there is a plethora of suitable papers available (Moccia and Chandroo 2003). Within his review, one can find many discussions where neurobiological literature pertaining to consciousness and pain perception in humans are utilized as if they were well-established facts, whereas the actual research behind those subjects or 'facts' are often hypothetical, preliminary or controversial. But it is our hope that his foundation paper, in addition to this current review, will help to open up the meaningful and critical dialogue relevant to a better understanding of consciousness and pain perception in fish.

\section{The neocortex and the neural correlates of consciousness}

The neocortex is a heterogeneous, laminated brain structure that comprises much of the cerebral cortex in humans (Nieuwenhuys 1994). The neocortex allows for sophisticated sensory processing, motor functions and is associated with distinctive human cognitive abilities. The role of cortical and noncortical brain structures in the generation of conscious states in humans has been the subject of intense debate and study (Kanwisher 2001; Baars 2002). We interpret the central thesis in Rose (2002) to be that conscious animals have a neocortex and animals without a neocortex, such as fishes, are by default, incapable of consciousness. The information presented in Rose (2002) pertaining to nociception, fear and learning processes in fish are eventually tied to the central theme that the neocortex is a prerequisite for any of these processes to 'reach' consciousness. Edelman and Tononi (2000), a reference source cited frequently within Rose (2002), states that, 'Many neuroscientists have emphasized particular neural structures whose activity correlates with conscious experiences. It is not surprising that different neuroscientists end up favouring different structures. As we shall see in a number of cases, it is likely that the workings of each structure may contribute to consciousness, but it is a mistake to expect that pinpointing particular locations in the brain or understanding intrinsic properties of particular neurons themselves, will explain why their activity does or does not contribute to conscious experience. Such an expectation is a prime example of a category error, in the specific sense of ascribing to things properties they cannot have' (Edelman and Tononi 2000, p. 19). Rose clearly, and for good reason favours the human neocortex as the structure of choice when it comes to attributing consciousness to a particular brain region, and suggests that specific areas of the neocortex are crucial for consciousness to occur (Rose 2002, p. 31). In order to support this claim, Rose primarily presents 'global workspace' theory (Baars 2002) or the 'dynamic core hypothesis' of Edelman and Tononi (2000), as well as evidence from clinical studies of humans afflicted with chronic vegetative states due to catastrophic brain injury (Laureys et al. 2000).

Although providing a brief, generalized description, Rose never sufficiently explains how, or why, the neocortex is thought to be responsible for consciousness, and careful examination of the neural-based theories of consciousness yields a viewpoint that does not necessarily support his arguments. Edelman and Tononi (2000), Laureys et al. (2000) and other work cited within Rose (2002) commonly implicate the thalamocortical system, and not the neocortex per se as the essential neural substrate required for consciousness. This is not a trivial detail, because as we explain later, it is precisely this interpretational difference that permits valid, alternative suggestions with regard to the neural requirements and evolutionary history of neural systems hypothesized to support consciousness. Theories proposed by Edelman and Tononi (2000) or tested by Laureys et al. (2000), account for the fundamental properties of consciousness by linking them to a particular type of neuronal process found primarily within the thalamocortical system (Tononi and Edelman 1998). The neuronal process within the thalamocortical system that may account for key properties of consciousness, is essentially described as the widespread integration of differentiated brain areas or functions (Baars 2002). A key tenet of the dynamic core hypothesis or other theories describing related neuronal processes, is that consciousness is 'generated' by a neural process per se, and as such is not accurately characterized as a specific thing or a location (Tononi and Edelman 1998). Therefore, if a nervous system has the appropriate characteristics that can support this process in theory, then it is appropriate, from a neurobiological perspective, to consider that this nervous system has the potential to 'generate' consciousness. Depending upon which hypothesis one ascribes to, the appropriate neuronal characteristics could include a variable level of complexity 
that reflects the interplay between functional segregation and integration within a neural system (Tononi et al. 1994), the level of degeneracy or redundancy within a neural system (Tononi et al. 1999), the process of neural signal re-entry (Sporns et al. 1991), particular thalamic functions (Llinás et al. 1998), specific neural activity synchronized at a particular gamma frequency (Sewards and Sewards 2001) and other features exhibited by vertebrate brains (Zeman 2001). It seems that Rose (2002) offers no cyto-architectural or neurophysiological data on the forebrain of fishes that can be used to argue whether or not this brain region can support any of the neuronal process mentioned above. Without this analysis, Rose's conclusion that, 'It is a neurophysiological impossibility for fish to have consciousness', is at best, unsubstantiated. Instead of a data-based or theoretical analysis, Rose relies on the belief that the fish brain is well understood and thus it is highly implausible that it could support consciousness (Rose 2002, p. 24). The argument suggests that fish forebrains have 'diminutive' dimensions (Rose 2002, p. 10), or 'poor differentiation' (Rose 2002, p. 28). Perplexingly, the discussion presented in Rose (2002) that does incorporate a selection of primary literature concerning the central nervous system of fishes is focused on the spinal cord and brain stem (Rose 2002, pp. 9-10, 22-23). Preliminary investigations into fish neurobiology suggest that adequate information currently exists to equally include or exclude the fish forebrain from having the capacity to support consciousness as defined by contemporary theory (Chandroo et al. 2004). Thus, the question of whether or not the nervous system of fish permits consciousness, from a purely neurobiological perspective, remains a very open question in our opinion. Innovative application of brain imaging techniques (Baars 2002) to fish species may provide new insights into the function of the fish telencephalon as it relates to neural processes associated with conscious states.

Many arguments within Rose (2002) rely upon controversial or untested interpretations of the neural-based theories of consciousness. In explaining why the neocortex exclusively is critical for consciousness, Rose asserts that there is clear and extensive evidence demonstrating that the human neocortex satisfies several, essential 'functional criteria', namely its unique structural features, that permit the existence of 'widely distributed brain activity that is simultaneously diverse, temporally coordinated and of high informational complexity' (Rose 2002, p. 7). However, it seems this argument is entirely circular: the human neocortex satisfies the critical, 'functional criteria' for consciousness, because the 'functional criteria' for consciousness are directly derived from the anatomy of the human neocortex. The majority of studies examining the neural correlates of consciousness do not support Rose's claim that 'the neurological basis of human consciousness is becoming increasingly well understood and is known to depend on functions of the neocortex' (Rose 2002, p. 31), or that 'the fundamental neural requirements for pain and suffering are now known' (Rose 2002, p. 33) (Damasio 1998; Llinás et al. 1998; Searle 1998; Tononi and Edelman 1998; Searle 2000; Jack and Shallice 2001; Kanwisher 2001; Parvizi and Damasio 2001; Zeman 2001; Baars 2002). Exactly how or why certain brain areas are associated with consciousness or pain perception is still largely controversial (Block 2001; Dennett 2001), and explanations for the associations are not by any means exclusive to a single theory or particular brain region (Sewards and Sewards 2000; John 2001). Rose reports that consciousness 'requires structurally differentiated neocortical regions with great numbers of exactly interconnected neurons' (Rose 2002, p. 24), and that the type of neocortex most essential to consciousness, i.e. the non-sensory association cortex, comprises the vast majority of the human cerebral cortex' (Rose 2002, p. 7, 31). However, the dynamic core hypothesis as proposed by Tononi and Edelman (1998), and cited by Rose to defend his argument, actually reports that the term dynamic core deliberately does not refer to a unique, invariant set of brain areas and that the core may change in composition over time. The dynamic core is also not necessarily restricted to the thalamocortical system, which is an important concept. Tononi and Edelman (1998) state that as neural participation in the dynamic core depends upon shifting functional connectivity among groups of neurones, rather than on anatomical proximity, the composition of the core can transcend traditional anatomical boundaries. As Rose equates consciousness exclusively with the neocortex, we suggest that his use of theoretical neurobiology is misleading and may be unsuitable for comparative assessment of fish brain function.

In other attempts to single out the neocortex as the exclusive structure enabling consciousness, Rose uses the literature to dissect the thalamocortical 
system (Rose 2002, p. 7, 13, 18). This is carried out to distinguish the neocortex from subcortical brain areas, implying that it is not significant that many animals have strikingly similar subcortical brain anatomy and function because they are not especially essential for the generation of consciousness. For example, Rose states that 'these [neocortical] structures and functional features are not present in subcortical regions of the brain, which is probably the main reason that activity confined to subcortical brain systems can't support consciousness' (Rose 2002, p. 7). He also states that '...consciousness also requires the operation of subcortical support systems such as the brainstem reticular formation and the thalamus, that enable a working condition of the cortex. However, in the absence of cortical operations, activity limited to these subcortical systems cannot generate consciousness' (Rose 2002, pp. 67). Although Rose cites data that reasonably support his claims, we again find that other variations in interpretation exist. Contemporary studies on the neural correlates of consciousness does not seem to support the suggestion that the thalamus behaves as a 'support system', so that the neocortex is enabled to generate consciousness. In fact, the role of subcortical activity within the human thalamocortical system is sometimes deemed as just as important for the 'generation' of consciousness per se, as is neocortical function (Llinás et al. 1998). In addition, Rose (2002), cites other works (Tononi and Edelman 1998; Edelman and Tononi 2000; Laureys et al. 2000) and commentary that just as clearly suggests that consciousness is more accurately described as a global functioning state of the brain, rather than a function of neocortical activity alone.

To further support his suggestion that the neocortex is the exclusive domain of consciousness, Rose extends a defence to include clinical studies pertaining to human patients in chronic, vegetative states. Rose describes a clinical condition that is intended to demonstrate that damage to the human neocortex renders a person vegetative and nonconscious (Rose 2002; pp. 13-14, 21), thus the neocortex must be responsible for any type of conscious state. This is a reasonable interpretation and hypothesis. However, there are three points of information that illustrate a possible contradiction in this logic. The first is that Rose fails to report that all of his examples refer to a pathological condition that radically affects the cerebral cortex in a way that also compromises thalamocortical activity. That is, thalamocortical activity and neocortical function are confounded in Rose's analysis. Secondly, Rose only reports cases of the persistent vegetative condition in which damage has occurred mostly to the neocortex, which permits an argument that the neocortex and not subcortical regions are therefore responsible for consciousness. However, vegetative patients demonstrating near-normal cortical metabolic rates (i.e. preserved cerebral function), but with damaged thalamic nuclei, have been documented (Schiff et al. 2002). Our last point refers to the effect of restricted neocortical lesions on consciousness. Edelman and Tononi (2000, p. 54) readily point out that 'despite occasional claims to the contrary, it has never been conclusively shown that a lesion of a restricted portion of the cerebral cortex leads to unconsciousness...no single area seems to hold the key to consciousness'. In fact, the only localized brain lesion that results in loss of consciousness typically affects the reticular activating system, a non-cortical structure found in all vertebrates. Again, it seems risky to equate consciousness as the exclusive domain of the neocortex.

Rose constructs several concise arguments contrasting the neurobiology of humans with that of other animals, and it therefore seems reasonable to suggest that the human forebrain is both quantitatively and qualitatively different on most accounts that matter to consciousness. These arguments suggest a unique, causative link between the physical size of the human neocortex, human intelligence and the fundamental aspects of brain organization that are supposedly specific to laminated mammalian brains. Rose emphasizes that it is not just the presence of the neocortex that is critical to consciousness, but a massive amount of neocortical expansion is also required (Rose 2002, p. 7, 10). And it continues that this massive neocortical expansion has allowed for the development of certain anatomical and cognitive traits that are distinctly human, including the lateralization of functions between the cerebral hemispheres (Rose 2002, p. 13), or the ability to have a psychological capacity (Rose 2002, p. 3). While there is no dispute that humans possess greatly expanded mental capacities that are associated with our brain structure, the application of this data to the analogous question of mental capacity in fish and other animals seems biased in our view. Consider the assertion that a massive expansion of neocortex must be present in order for consciousness to occur, and that this neurological requirement is essential for a psychological capacity (Rose 2002, p. 3, 29, 
31). Edelman and Tononi (2000, p. 53) mention several clinical reports of human patients, who have lost (via surgery), or failed to develop, massive neocortical expansion, and yet have reasonably normal cognitive abilities and intelligence quotients. These anomalous observations, considered together with the reality that there is still little consensus among neurobiologists as to how consciousness is actually 'generated', point to the fact that modern theories of the neural correlates of consciousness are still just tentative explanations. But, we suppose that this is fair enough at such an early stage of debate of these complex paradigms. But Rose subtly portrays some of these theories as biological facts, without explaining the necessary caveats and underlying assumptions inherent in these theories. The observation is made that the majority of the activity in our extensive brain matter is 'unavailable' to our conscious awareness, and therefore, for an organism like a fish (i.e. having a smaller, less complex brain), it is entirely logical that none of their brain activity could be dedicated to conscious experience (Rose 2002, p. 15). However, as pointed out by Griffin (1998), the implied assumption within Rose's reasoning is that the proportion of conscious to unconscious activity must be even smaller in the non-human, animal brain. Rose fails to provide any neurobiological data that could justify that assumption (perhaps because such data does not exist?), yet studies of human subjects whose brain development or size had been limited also do not support that premise (Edelman and Tononi 2000). As Griffin (1998) remarks, 'perhaps only in "megabrains" is most of the information processing unconscious...insofar as simple conscious thinking is effective and adaptive, it may be one of the important functions a central nervous system.' Rose states that 'expansion of the cerebral hemispheres has also allowed lateralized functions of the two cerebral hemispheres...' and alludes to the fact that certain lateralized cognitive functions are manifestations of higher-order consciousness (Rose 2002, p. 13). The idea that massive cortical expansion is necessary for the lateralization of cerebral functions seems simplistic. A brain is considered to be cerebrally lateralized if one hemisphere performs a different set of cognitive functions, or is anatomically distinguishable from the other (Bisazza et al. 1998). There are many studies that show lateralization of cognitive functions involved in social interactions, learning and perceptual categorization occurs in many vertebrate species, including fishes (Bisazza et al. 1998; Vallortigara 2000), and that fundamental similarities between the cerebral structures of all vertebrates exist. Clearly, the association between absolute brain size, brain organization, cognition and consciousness is not as clear-cut as Rose argues, and as such, his application of these concepts to the question of consciousness in fish is divisive.

\section{Convergence, homology and evolutionary psychology}

Rose (2002) emphasizes the impressive differences between the brain structure of fish and humans. However, when the analysis of brain structure and function is extended to all major vertebrate groups, the vertebrate central nervous system appears to have had a rather conservative evolution. The structural or functional differences between species can be accurately described as specialized adaptations within a consistent overall organization (Butler and Hodos 1996). We believe that Rose would consider the neural substrate for 'primary' or 'extended' consciousness to be a specialized adaptation exclusive to humans (Rose 2002, p. 6). However, notably absent from Rose's evolutionary perspective is a consideration of the process of convergent evolution. Convergent evolution appears at all level of biological organization, and is a process by which similarity between unrelated species occurs because of adaptation to similar environmental pressures (Wray 2002). Convergence can occur on a functional level without the complete convergence of underlying structural elements.

If Rose's analysis is correct, and human neocortical structure is the only neural substrate capable of producing consciousness beyond a rudimentary extent, then different neuroanatomical arrangements of the forebrain should result in animals with very dissimilar cognitive capabilities and little or no manifestations of primary or extended consciousness. Marino (2002) provides data and analysis that test this hypothesis and compares primate and cetacean biology, and describes aspects of their independent evolutionary history such as adaptations to drastically different physical environments (terrestrial vs. aquatic), as well as pronounced differences in body shape and physiology. He also demonstrates that cetacean forebrains are organized in fundamentally different patterns from that observed in the brains of primates, to the extent 
that cetaceans can be considered as having a completely different mode of cortical elaboration. Interestingly, cetaceans demonstrate cognitive abilities that are elsewhere only found in humans or the great apes - abilities that were traditionally assumed to be solely the result of human neocortical structure and function. Marino (2002) clearly shows that cognitive and behavioural convergence can occur, even in the face of profound neuroanatomical divergence. The cognitive abilities shown by cetaceans are also widely accepted as manifestations of primary or extended consciousness (Marino 2002). As cetaceans do not show the 'extensive frontal and parietal lobe of neocortex', or, 'expansive, specialized six-layered type of cortex' that Rose suggests is the most important brain level requirement for conscious awareness and other cognitive abilities (Rose 2002, p. 32), it would seem that his argument can be challenged at the conceptual level. Namely, the specific neurobiological way that a species arrives at a functional solution is not the only level by which to understand it, especially when comparing disparate species of animals. Thus, the neural substrate of consciousness does not necessarily invoke the involvement of a six-layered laminar structure, but instead needs to fulfil some other aspect of a specific neural process, such as those described within Searle (2000) or Baars (2002). An examination of brain structure and function between distantly related species, which demonstrate cognitive adaptations characteristic of primary consciousness may further reveal the nature of these neural processes.

We review the work of Marino (2002) as one example of how different species have evolved alternate mechanisms to increase their brain mass and function. Enlargement and elaboration of the forebrain has independently occurred multiple times within different lineages of vertebrates, within fish and mammalian species (Butler and Hodos 1996). Contrary to the suggestions found in Rose (2002), the forebrains of some fish do, in fact, represent complex, elaborated structures within the vertebrate radiation. Anatomical features and functions of the fish forebrain may be homologous or convergently related to similar structures and functions in mammals. Despite evidence to the contrary, Rose strongly denies that any functional homologies (especially limbic brain regions implicated in conscious states) exist in the fish brain (Rose 2002, p. 28). His reasoning is that such homologies are simply structural, and therefore it is misleading to ascribe a comparable function with those structures. He also favours the thesis that functional equivalency for any limbic structure found both in fish and mammals is impossible, because fish do not have a neocortex. We find the first reason perplexing, because the majority of limbic structures in the fish brain have not been defined simply because their structures have been conserved during evolution, but specifically because they have similar physiological and behavioural function as in other vertebrates (López et al. 2000; Portavella et al. 2002). It is to be expected that limbic structures will have modified interaction and function within the fish and human cerebrum because those cerebral structures must mediate different cognitive tasks. However, the assertion that functional equivalency of limbic structures is impossible because fish do not have a neocortex implies that the influence of the neocortex on other brain structures is to make those brain structures functionally dissimilar. That conclusion seems unsupported by others (Butler and Hodos 1996), and demonstrates an anatomical bias in Rose's reasoning. Many limbic brain structures found in mammals have functionally similar counterparts within the brains of fish (reviewed in Chandroo et al. 2004).

Although Rose suggests that some mammals have a primary, rudimentary form of consciousness (because their cerebral hemispheres show limited neocortical structure), he gives little indication as to what primary consciousness actually implies in terms of an animal's psychological capacity. This is significant, because delineating the exact nature of the psychological differences between humans and other species is necessary if we are to develop a valid understanding of how and why a psychological capacity arose during vertebrate evolution. In the discussion that appears to address the question of psychological capacities within vertebrate phyla, it seems that Rose adopts an overly anthropocentric view (Rose 2002, p. 3). That is, any psychological capacity that can be observed in humans is assumed to be uniquely human, so any suggestion that animals have similar mental capacities can be immediately dismissed as anthropomorphic. Rose goes on to describe unique aspects of human psychology, such as our capability for creativity including art, science and the existence of religious beliefs. He uses these specific human abilities as examples that would make us seem so distinct, that it would be highly inappropriate and misleading to project any human-like psychological characteristic 
whatsoever onto other species. And this may be a reasonable premise. Yet, he then presents examples of electroreception and signalling by electric fish, or echolocation in bats and dolphins as unique capacities endowed to these animals that have no equal counterpart in humans. This also sets the stage for Rose's opinion of consciousness (and therefore psychological abilities as well), being likewise a unique capacity, has no counterpart in most animals.

The opinions expressed by Rose reflect one of two competing philosophical paradigms that characterize the approach to understanding the evolution of the human mind. These approaches are termed the Cartesian and Darwinian perspectives (Gibson 2002). Rose apparently ascribes to the Cartesian perspective, which postulates that human and animal minds are separated by major qualitative differences in mental abilities. The Darwinian perspective postulates more continuity between animal and human mental capacities; in other words, the differences between the animal and human mind is more a matter of degree rather than kind (Gibson 2002). As empirical data from comparative neurobiology and ethological studies have revealed limitations in the explanatory value of the Cartesian perspective for a number biological phenomenon, including the learning behaviour of animals, we tend to favour the later conceptual approach to understanding the animal mind.

Animal behaviour research suggests that rudiments of most human cognitive abilities also exist in great apes (reviewed in Gibson 2002). These cognitive abilities, many of which cannot be explained by traditional associative theory, are thought to occur through a process that is termed 'mental construction'. Mental construction refers to the brain's ability to generate a representation of internal and external events. These mental representations function as a predictive model of the environment, allowing for the construction of new knowledge. Utilization of this knowledge permits an animal to express novel, adaptive behaviour (Topál and Csányi 1999; Edelman and Tononi 2000; Gibson 2002). As cognitive abilities that are better explained by means of mental construction have been shown to exist in both animal and human minds, it has been hypothesized that enhanced human mental constructional capacities underlie human creativity and mental flexibility (Gibson 2002). According to Gibson (2002), the improved information processing abilities of the enlarged human brain endows our species with greater abilities to break concepts and actions into fine component parts, and to combine these differentiated components into higher order behavioural and mental constructs. It is these mental construction capacities that serve as a common foundation for the wide-ranging behavioural domains in which human intellectual abilities resemble and improve on those of other primates (Gibson 2002). If we assume that the capacity for mental construction is associated with a psychological capacity, or is characteristic of primary consciousness, then the observation that mental construction occurs in a variety of distantly related vertebrate taxa would argue that basic psychological capacities, as well as primary consciousness, is phylogenetically old. We suggest that the 'building blocks' for psychological capacity and primary consciousness may exist within certain fundamental neural attributes and processes shared by many vertebrate animals (Chandroo et al. 2004). The hypothesis that the ability for mental construction was associated with the emergence of primary consciousness in animal species, has also been proposed within tenets of certain neural theories of consciousness (Edelman and Tononi 2000). Rose (2002) contains no data that enable us to determine whether or not mental representation occurs in fish species, or that mental constructs of a lesser complexity are not associated with primary consciousness. The issue of mental constructs in fish has been reviewed in Chandroo et al. (2004), and we suggest that such a cognitive ability is indeed feasible for fish species.

\section{Pain perception and brain structure}

Following the initial discussion on the neocortex and consciousness, Rose (2002) gives a review of pain perception in humans, with an eventual application to the question of pain perception in fish. Rose begins the pain perception review with an analysis of nociception and pain in humans, appropriately making the distinction between transduction of tissue trauma into neural signals (i.e. nociception), and central registration of nociception (i.e. the processes involved in consciously experiencing pain). Rose states that "pain is a psychological experience that is separate from behavioural reactions to injurious stimuli' (Rose 2002, p. 15) and we accept this definition. However, for Rose, subcortical events (e.g. behavioural reactions, and presumably their accompanying autonomic nervous system processes) involved with nociception have nothing 
to do with the conscious awareness of pain, because, '...the behavioural displays related to noxious stimuli or emotion in humans, as in other animals, are stereotyped, automatic behavioural programs controlled by lower levels of the central nervous system.' (Rose 2002, p. 17) The supporting evidence given for these suggestions is that one can observe 'pain-like' responses in humans and animals with neocortical damage or impaired nociceptive transmission preventing signals from reaching the neocortex. Eventually, Rose's argument reduces to the premise that lack of a neocortex essentially means that there can be no pain perception.

Conceptually, Rose's view of the mechanisms of pain perception is questionable. For example, consider his reasoning behind the claim, pain is a psychological experience that is separate from behavioural reactions to injurious stimuli.' Contrary to Rose's assertions, the arousal of the subcortical autonomic nervous system (and the associated defensive behaviour response) plays a paramount role in creating the psychological experience of pain, and is not a functionally separate entity in normal humans (Chapman and Nakamura 1999; Saper 2002; Craig 2003). Although it is perfectly acceptable from an instructive point of view to subdivide pain perception into anatomic categories (e.g. cortical and subcortical), this dichotomous portrayal is ultimately limited as far as gaining an accurate understanding of human pain perception, as well as in its application to the question of pain perception in other species (Price et al. 2002). Although Rose's conception of the neural mechanisms of pain perception can be demonstrated with humans or animals with damaged, impaired central nervous systems, one must ask - how does this relate to pain perception in normal human subjects, and what, if anything, does it say about the evolution of pain perception in lower vertebrates?

Pain perception in humans is not simply a series of reflexive behavioural responses accompanied by a non-functional, distinctly separate, cortical-mediated subjective feeling. Rose does not acknowledge this concept, and as such, his arguments reflect a dualistic perspective to pain perception. In addition to his 'consciousness as a computer monitor' analogy in which consciousness is portrayed as a passive window in which brain processes may come to awareness (Rose 2002, p. 15), Rose cites a passage from LeDoux (1996) that states, 'the brain states and bodily responses are the fundamental facts of an emotion, and the conscious feelings are the frills that have added icing to the emotional cake' (Rose 2002, p. 26). Rose seems to suggest that the conscious, subjective aspects of pain perception have no tangible or adaptive function, but are epiphenomenal. The hypothesis that pain is a sensory end product of a passive information transmission process has largely been rejected among pain researchers (Chapman and Nakamura 1999). It is curious how Rose can present a dualistic perspective for a conscious state because the work he cites in order to report that the neocortex is the exclusive structure responsible for consciousness explicitly requires the underlying assumption that consciousness is not epiphenomenal, but functional and adaptive (Edelman and Tononi 2000). Although Rose argues that an evolutionary perspective is necessary for examining the question of pain perception in fish species, his apparent dualistic view of pain perception is largely incompatible with an evolutionary account for its existence.

Although it is easy to gain the perception from Rose (2002) that it is becoming very clear as to why and how the neocortex is responsible for pain perception, this portrayal does not fully reflect the current scientific literature (Besson 1999; Chapman and Nakamura 1999; Treede et al. 1999). In brain imaging studies, only the anterior cingulate gyrus (a 'limbic' system brain component) has demonstrated a consistent response during the conscious experience of pain in humans (Derbyshire et al. 1997). Interestingly, the anterior cingulate gyrus does not show the classical neocortical, six-layered structure, but its cellular conformation resembles a five-layered structure, with other distinctions (Nimchinsky et al. 1997). Although we could argue that this neurophysiological fact casts doubt on Rose's claim that only specific, specialized neocortex can be responsible for conscious perception of pain in humans or animals, we instead suggest that this line of reasoning in general is fruitless in any event. Tononi and Edelman (1998) and others (Baars 2002) advocate that it is not any specific structure (e.g. a cortex with five or six layers) or particular location that is associated with the generation of consciousness, but rather, it is the type of neuronal activity per se that the structure participates in that is critical for the generation of consciousness. While Rose's review brings forth some pertinent issues needed to assess the question of pain perception in fish, his interpretations of the neurobiological underpinnings of human pain perception combined with a lack of primary literature concerning fish neurobiology and behaviour preclude him from 
making any firm conclusions on the existence of pain perception in those species.

We address the question of pain perception in fish by first accepting the assumption that it is unlikely that the conscious perception of pain evolved to simply guide reactions to noxious events, or to provide an experiential dimension to accompany reflexes, but rather it allowed an organism to discriminate their environment in ways that permitted adaptive and flexible behaviour (Chandroo et al. 2004). The neural systems involved in nociception and pain perception, and the cognitive processes resulting in flexible behaviour function, probably evolved as an interactive dynamic system within the central nervous system (Chapman and Nakamura 1999). The creation of a subjective feeling of pain is arguably a complex affair, including spinal, brainstem, thalamic and cerebral structures, as well as essential autonomic nervous system feedback (Willis and Westlund 1997; Chapman and Nakamura 1999; Saper 2002). Spinal, thalamic and forebrain system interdevelopment has been a primary mode of central nervous system evolution in vertebrate species (Kevetter and Willis 1984; Butler 1994). Accordingly, it is reasonable to suggest that the evolution of pain perception should show concomitant developments in all those neural structures, and these developments should be reflected within an animal's cognitive abilities. Therefore, a pertinent question to ask when attempting to determine the origins of pain perception during vertebrate evolution is when, and in what form, does this unified, integrative system appear? How do we recognize when nociceptive signals arrive at the forebrain and participate in the cognitive processes characteristic of conscious, adaptive behaviour?

Work performed by Sneddon (2003) and Sneddon et al. (2003) are a step in the right direction to provide insights for the questions we pose. Sneddon et al. (2003), Sneddon (2003) and Ide and Hoffmann (2002) report experimental studies that contribute significantly to the question of nociception and pain perception in fish species. Their studies contain data that describes the function of peripheral and central nervous system structures involved in nociception, and the correlation of nociceptor activation with autonomic and behavioural responses. Sneddon (2003) and Sneddon et al. (2003) interpret their work within an ethological and physiological framework that allows them to conclude that their results fulfil 'the criteria' for demonstrating pain in animals, and that accordingly, fish can perceive pain. After defining pain in humans as an 'unpleasant sensory and emotional experience associated with actual or potential tissue damage,' Sneddon et al. (2003) state that, it is impossible to truly know whether an animal has an emotion because we cannot measure emotion directly. Therefore, emotion does not feature in the definition of pain in animals.' They further describe their criterion for pain perception by stating '...if a noxious event has sufficient adverse effects on behaviour and physiology in an animal, and this experience is painful in humans, then it is likely to be painful in the animal' (Sneddon et al. 2003, p. 2). The criterion that Sneddon et al. (2003) describe is adequate for assessing clinical pain responses in animals whose pain system is well understood (e.g. domestic mammals). However, when this criterion is used for the purpose of elucidating the existence of pain perception in animals that are significantly less understood (e.g. fish), subjective leaps in the interpretation of the results are required to come to firm conclusions on the issue.

Sneddon (2003) shows that injection of a known noxious substance into peripheral tissue innervated by nociceptors, causes several physiological and behavioural reactions not found in control fish, such as a significantly increased respiratory rate, a delay in the time it takes for fish to resume feeding, and rocking and rubbing behaviour. Sneddon (2003) also shows that these induced responses greatly diminish when morphine is administered intramuscularly. Clearly, the responses of fish to noxious stimuli and morphine require the integration of peripheral and central nervous system structures. However, we must ask what relevance these results have to the question of actual pain perception. Sneddon (2003) first attempts to address that question by suggesting that rocking behaviour of fish may be similar to the rocking behaviour shown by primates and zoo animals which are exhibiting signs of poor welfare. Beyond the superficial similarity, this suggestion is not further supported by any other data presented. There is no effort made to include or exclude alternative accounts for the rocking behaviour that do not require the involvement of consciousness for its explanation (e.g. the effect of metabolic alterations caused by prolonged hyperventilation, subsequent swim bladder or equilibrium reactions, the effect of corticosteroid and catecholamine responses, as well as the effect of trigeminal nerve input on brainstem controlled 
motor behaviour). In addition, Sneddon makes no attempt to explain or compare the putative proximate and ultimate causes for rocking behaviour in primates and fish. Such a comparison would need to address the question of why rocking, primarily a primate behaviour elicited by diverse aetiologies (Nash et al. 1999), should have the same cognitive underpinnings and social function in a rainbow trout. This seems highly unlikely. Similarly, Sneddon compares the rubbing behaviour of fish to the act of rubbing an injured area to ameliorate the intensity of pain as observed in humans and mammals. She also states that rubbing behaviour and rocking, may be potential pain-coping strategies in fish (Sneddon 2003, p. 7). Again, the data or analysis needed to confirm these suggestions are absent, and as such, she cannot exclude noncognitive or non-conscious explanations for such behaviour. Although Sneddon et al. (2003) have described the composition and response characteristics of fish nociceptors, they have not presented any anatomical or physiological data to suggest that afferent inhibition occurs in fish species (i.e. the process behind pain amelioration via rubbing).

Sneddon suggests that the rocking and rubbing behaviour of fish is complex in nature, and therefore higher processing is involved (Sneddon 2003, p. 8). This leads her to conclude that fish can perceive pain. In light of the critique we have presented, we suggest that this interpretation is premature. Based on the information presented in Sneddon (2003), labelling such behaviour as complex is a simple value judgment. Even if nociception-induced rocking and rubbing behaviour was complex, the assumption that complex behaviour per se must be indicative of 'higher processing' is incorrect (Shettleworth 2001). Furthermore, the concept that higher cognitive processing is synonymous with conscious cognition is misleading (Bargh and Ferguson 2000). We suggest that alternative criteria should be used to determine if behavioural responses are reflective of pain perception in fish. The behaviour meeting this criterion should permit the distinction between responses that involve the integration of brain structures that are hypothesized to be involved in the process of conscious cognition and of those that do not. Such behaviour might include those that are observed as the result of interactive or declarative learning processes (Chandroo et al. 2004).

Other studies that use autonomic and behavioural responses of fish to assess whether they are conscious have been performed by Cabanac (1999). Based on empirical studies focused on autonomic physiological responses, Cabanac (1999), as well as Cabanac and Cabanac (2000) suggest that consciousness and emotion evolved with the appearance of amphibians or reptiles. They reason that as fish do not show autonomic responses to 'emotional stress' (i.e. tachycardia or behavioural fever) in the same way terrestrial animals do, they probably are not conscious. There are factual and conceptual errors with this argument. Fish do in fact exhibit tachycardia in response to circumstances that one might expect a similar emotional response to occur in mammals (Höjesjö et al. 1999). Cabanac's hypothesis does not account for the observation that emotionally influenced autonomic responses are associated with behaviour that is relevant to surviving species-specific environmental challenges. For example, an animal that evolved a 'freeze' strategy to a predation threat likely have altered autonomic responses to an animal that evolved a 'flight response'. The autonomic response to specific stimuli can even change with age within single species (Höjesjö et al. 1999). The expectation that certain autonomic or behavioural responses should be similarly associated with specific emotions among diverse vertebrate groups is unwarranted at this time, although it is a useful paradigm to examine. Future work should aim to account for the mechanism by which behavioural or autonomic responses are seated within cerebral processes that may be associated with the existence of conscious states.

\section{Cognition and behaviour in fishes}

Rose has reviewed a subset of the extensive contemporary knowledge available on the neurobehavioural nature of fish. The primary literature concerning the neurobiological features and learning behaviour of fish and other non-human vertebrates is somewhat weakly presented in Rose (2002), and it undoubtedly is the reason for many of the arguments that support his conclusions. He argues that '...most behaviour of fishes is not dependent greatly on learning...' (Rose 2002, p. 8), '...instrumental and Pavlovian conditioning are forms of associative, implicit learning that occur in fishes... as cases of implicit learning, they operate without awareness...' (Rose 2002, p. 27), '...nothing about the behaviour of a fish requires a capacity for conscious awareness for its explanation' (Rose 
2002, p. 24), '...avoidance conditioning occurs unconsciously and is not evidence of awareness of pain or any other experience' (Rose 2002, p. 29), '... in fishes, our brainstem-spinal systems are adequate for generation of overt reactions' (Rose 2002, p. 25) and '...behavioural specialization in fishes is associated with expanded brainstem as opposed to cerebral hemisphere development' (Rose 2002, p. 10). All of those statements are misleading in our opinion.

Rose claims that fish behaviour in general is not greatly dependent on learning, except for the initial development of species-typical behaviour (Rose 2002 , p. 9). The idea that fish behaviour is dominated by pre-programmed, invariant responses to the environment, and that the main significance of learning is to 'prime' the development of such responses is perhaps more reflective of a historical view of fish behaviour rather than of any current data (Laland et al. 2003). The relevance of learning to fish behaviour at various life-history stages has been increasingly investigated over the past decade (Overmier and Hollis 1990; Kieffer and Colgan 1992; Csányi and Dóka 1993; Bshary et al. 2002). The learning processes shown by fish include observational (McGregor et al. 2001), interactive (Topál and Csányi 1999), Pavlovian (Hollis 1984) and avoidance (Zerbolio and Royalty 1983). Current work shows that learning processes demonstrated by fish are multifaceted phenomenon that have clear fitness implications to fish species at various developmental stages (Brown and Laland 2003; Griffiths 2003; Hoare and Krause 2003; Kelley and Magurran 2003; Odling-Smee and Braithwaite 2003).

Rose presents Pavlovian (i.e. classical conditioning) and avoidance learning exclusively to describe learning processes in fish. He further defines Pavlovian and avoidance learning as examples of implicit learning. The argument then goes on to state that implicit learning has no relationship to any conscious process, and must therefore occur without conscious awareness. The suggestion that implicit learning has no relationship to higher order cognition, or is inherently an unconscious process is a concept that is not universally accepted because there is empirical data that show otherwise (Maren 2001; Lovibond and Shanks 2002). Similarly, the suggestion that avoidance learning in fish species is purely a form of implicit learning with no other significance to cognition is incorrect according to Overmier and Hollis (1990). We suggest that observational, avoidance and interactive learning processes may require the formation of declarative memories. The relationship between learning processes demonstrated by fish, declarative memory and conscious cognition has been reviewed in Chandroo et al. (2004), and there is, in fact, an objective basis for suggesting that some fish behaviour is better explained within a theoretical framework that includes primary consciousness as a function of their nervous system. Given the fact that none of the learning processes we mention are considered in Rose (2002), his statement that 'nothing about a fish's behaviour could be conscious' seems unqualified and incomplete.

There are several arguments found within Rose (2002) that suggest that fish are unique among vertebrates in that their behavioural specialization is dependent heavily upon brainstem development, and that the fish cerebrum has little significance to their behaviour beyond olfaction, or to 'refine the expression' of brainstem functions (Rose 2002, p. 9). He further states that, 'the neurobehavioral evolution of fishes has resulted in a highly diversified array of species in which the essentials of neurobehavioral function are mediated mainly by a neural system below the cerebral hemispheres' (Rose 2002, p. 9). To support these claims, he suggests that fish function, learn and behave essentially normally (except for functions requiring olfaction, which Rose claims is processed entirely within their forebrain) after their cerebrum is ablated. The logic here is misleading. The concept that the fish cerebrum functions primarily as a 'smell brain' has been rejected by most comparative neurologists for some time (Echteler and Saidel 1981). Although Rose brings forth the valid notion that one function of the fish cerebrum is to modulate behavioural expression, he fails to acknowledge the implications of this function in terms of fish cognition. Ablation of the fish cerebrum does in fact impair learning and behaviour that are hypothesized to involve expectancies, complex spatial cognition, declarative memory or mental construction processes (Overmier and Hollis 1990; Broglio et al. 2003; Chandroo et al. 2004). Rose chooses to report only the learning and behavioural processes that do not require the involvement of such cognitive processes for their explanation, and distorts the definition of avoidance learning to suit his arguments. Rose's theoretical argument that evolution of the central nervous system of fishes has resulted in mainly brainstem 
development, and that their success as a species has little to do with cerebral modification is refuted by empirical evidence. All teleost fish have elaborate forebrains (Butler and Hodos 1996), and the degree of forebrain development is correlated with social behaviour, communication abilities and other environmental factors that may require integrative cognitive capacities (Kotrschal et al. 1998). Fish have evolved to exploit diverse environmental niches, and show concomitant development within all relevant brain areas. This brain development may consist of increases or decreases in brain stem, cerebellar, optic, olfactory, diencephalic and telencephalic structural mass or complexity (Kotrschal et al. 1998). The morphological changes that may comprise the representative brain for any given species are diverse, and contrary to Rose's generalized assumption, some phylogenetic radiations (e.g. actinoperygians) show a shift in brain mass from primary sensory areas towards higher order integration centres (Kotrschal et al. 1998). In addition, the integration of cerebellar, optic and telencephalic functions to produce cognitive responses to the environment may be similar in fish and mammals (Broglio et al. 2003). Although Rose briefly comments on the great morphological variation of fish brains and the putative functions of the fish cerebrum (Rose 2002, p. 9, 10), he fails to give a balanced account of the implication that such diversity and development have to cognitive functions, and the question of consciousness in fish species.

\section{Conclusions}

If the debate regarding the existence of sentience in fish is to have valid conclusions, the basis of the arguments must be made upon sound biological principles, taking into account all sources of relevant data. Our critique has demonstrated that the input of recent behavioural, neurological or physiological findings into the analysis can profoundly change the possible conclusions reached about the mental capacity of fishes. We have argued that if one adopts a Darwinian perspective to the study of animal minds, it is not simply a matter of more, less, or no neocortex present that permits the existence of a neural system that may support primary consciousness. A current limitation of theories describing the neural basis of consciousness in humans is that it essentially examines the intrinsic properties of complex neural systems, often without considering the question of how those characteristics arose during evolution (Tononi et al. 1994). If a neural process, whether its substrate is laminated or otherwise, allows some degree of mental construction to occur, and then it may be reasonable to suggest that those animals may have evolved primary consciousness. Autonomic and behavioural responses that are used to prove or refute the existence of conscious states in fish species need to be assessed for their involvement within integrative cognitive processes that are associated with mental construction, declarative memory or other possible indicators of primary consciousness. A sound assessment of the probability that conscious states occur in fish species will require expanded knowledge of their forebrain neuroanatomy, an understanding of how such structures mediate behavioural responses to environmental challenges and an analysis of that information within the context of contemporary theory on the evolution of consciousness.

\section{Acknowledgements}

We wish to thank the Ontario Ministry of Agriculture and Food for partial, financial support of this project. We also thank Dr Ian Duncan for his input to many of the issues discussed.

\section{References}

Baars, B.J. (2002) The conscious access hypothesis: origins and recent evidence. Trends in Cognitive Sciences 6, 47-52.

Bargh, J.A. and Ferguson, M.J. (2000) Beyond behaviorism: on the automaticity of higher mental processes. Psychological Bulletin 126, 925-945.

Besson, J.M. (1999) The neurobiology of pain. The Lancet 353, 1610-1615.

Bisazza, A., Rogers, L.J. and Vallortigara, G. (1998) The origins of cerebral asymmetry: a review of evidence of behavioural and brain lateralization in fishes, reptiles and amphibians. Neuroscience and Biobehavioral Reviews 22, 411-426.

Block, N. (2001) Paradox and cross purposes in recent work on consciousness. Cognition 79, 197-219.

Broglio, C., Rodriguez, F. and Salas, C. (2003) Spatial cognition and its neural basis in teleost fishes. Fish and Fisheries 4, 247-255.

Brown, C. and Laland, K.N. (2003) Social learning in fishes: a review. Fish and Fisheries 4, 280-288.

Bshary, R., Wickler, W. and Fricke, H. (2002) Fish cognition: a primate's eye view. Animal Cognition 5, $1-13$. 
Butler, A.B. (1994) The evolution of the dorsal pallium in the telencephalon of amniotes: cladistic analysis and a new hypothesis. Brain Research Reviews 19, 66-101.

Butler, A.B. and Hodos, W. (1996) Comparative Vertebrate Neuroanatomy. Wiley-Liss, Inc., New York, USA.

Cabanac, M. (1999) Emotion and Phylogeny. Japanese Journal of Physiology 49, 1-10.

Cabanac, A. and Cabanac, M. (2000) Heart rate response to gentle handling of frog and lizard. Behavioural Processes 52, 89-95.

Chandroo, K.P., Duncan, I.J.H. and Moccia, R.D. (2004) Can Fish Suffer? Perspectives on sentience, pain, fear and stress. Applied Animal Behaviour Science 86, 225250.

Chapman, C.R. and Nakamura, Y. (1999) A passion of the soul: an introduction to pain for consciousness researchers. Consciousness and Cognition 8, 391-422.

Craig, A.D. (2003) Pain mechanisms: labeled lines versus convergence in central processing. Annual Review of Neuroscience 26, 1-30.

Csányi, V. and Dóka, A. (1993) Learning interactions between prey and predator fish. Marine Behaviour and Physiology 23, 63-78.

Damasio, A.R. (1998) Investigating the biology of consciousness. Philosophical Transactions of the Royal Society of London: Series B 353, 1879-1882.

Dennett, D. (2001) Are we explaining consciousness yet? Cognition 79, 221-237.

Derbyshire, S.W.G., Jones, A.K.P., Gyulai, F., Clark, S., Townsend, D. and Firestone, L.L. (1997) Pain processing during three levels of noxious stimulation produces differential patterns of central activity. Pain 73, 431445.

Echteler, S.M. and Saidel, W.M. (1981) Forebrain connections in the goldfish support telencephalic homologies with land vertebrates. Science 212, 683-684.

Edelman, G.M. and Tononi, G. (2000) A Universe of Consciousness. Basic Books, New York, NY.

Gibson, K.R. (2002) Evolution of human intelligence: the roles of brain size and mental construction. Brain, Behavior and Evolution 59, 10-20.

Griffin, D.R. (1998) From cognition to consciousness. Animal Cognition 1, 3-16.

Griffiths, S.W. (2003) Learned recognition of conspecifics by fishes. Fish and Fisheries 4, 256-268.

Hoare, D.J. and Krause, J. (2003) Social organization, shoal structure and information transfer. Fish and Fisheries 4, 269-279.

Höjesjö, J., Johnsson, J.I. and Axelsson, M. (1999) Behavioural and heart rate responses to food limitation and predation risk: an experimental study on rainbow trout. Journal of Fish Biology 55, 1009-1019.

Hollis, K.L. (1984) The biological function of Pavlovian conditioning: the best defense is a good offence. Journal of Experimental Psychology, Animal Behavior Processes 10, 413-425.
Ide, L.M. and Hoffmann, A. (2002) Stressful and behavioral conditions that affect reversible cardiac arrest in the Nile tilapia, Oreochromis niloticus (Teleostei). Physiology and Behavior 75, 119-126.

Jack, A.I. and Shallice, T. (2001) Introspective physicalism as an approach to the science of consciousness. Cognition 79, 161-196.

John, E.R. (2001) A field theory of consciousness. Consciousness and Cognition 10, 184-213.

Kanwisher, N. (2001) Neural events and perceptual awareness. Cognition 79, 89-113.

Kelley, J.L. and Magurran, A.E. (2003) Learned predator recognition and antipredator responses in fishes. Fish and Fisheries 4, 216-226.

Kevetter, A.G. and Willis, W.D. (1984) Collateralization in the spinothalamic tract: new methodology to support or deny phylogenetic theories. Brain Research Reviews 7 , $1-14$.

Kieffer, J.D. and Colgan, P.W. (1992) The role of learning in fish behaviour. Reviews in Fish Biology and Fisheries 2 , 125-143.

Kotrschal, K., van Staaden, M.J. and Huber, R. (1998) Fish brains: evolution and environmental relationships. Reviews in Fish Biology and Fisheries 8, 373-408.

Laland, K.N., Brown, C. and Krause, J. (2003) Learning in fishes: from three-second memory to culture. Fish and Fisheries 4, 199-202.

Laureys, S., Faymonville, M.E., Luxen, A., Lamy, M., Franck, G. and Maquet, P. (2000) Restoration of thalamocortical connectivity after recovery from persistent vegetative state. The Lancet 355, 1790-1791.

LeDoux, J. (1996) The Emotional Brain. Simon and Schuster, New York.

Lindahl, B.I.B. (1997) Consciousness and biological evolution. Journal of Theoretical Biology 187, 613-629.

Llinás, R., Ribary, U., Contreras, D. and Pedroarena, C. (1998) The neuronal basis for consciousness. Philosophical Transactions of the Royal Society of London: Series B 353, 1841-1849.

López, J.C., Broglio, C., Rodríguez, F., Thinus-Blanc, C. and Salas, C. (2000) Reversal learning deficit in a spatial task but not in a cued one after telencephalic ablation in goldfish. Behavioural Brain Research 109, 91-98.

Lovibond, P.F. and Shanks, D.R. (2002) The role of awareness in Pavlovian conditioning: empirical evidence and theoretical implication. Journal of Experimental Psychology 28, 3-26.

Maren, S. (2001) Neurobiology of Pavlovian fear conditioning. Annual Review of Neuroscience 24, 897-931.

Marino, L. (2002) Convergence of complex cognitive abilities in cetaceans and primates. Brain, Behavior and Evolution 59, 21-32.

McGregor, P.K., Peake, T.M. and Lampe, H.M. (2001) Fighting fish Betta splendens extract relative information from apparent interactions: what happens when what 
you see is not what you get. Animal Behaviour 62, 1059-1065.

Moccia, R.D.M. and Chandroo, K.P. (2003) Aspects of animal welfare and aquaculture - a compendium of selected literature. Aquaculture Centre, University of Guelph, Guelph, Canada (http://www.aps.uoguelph.ca/ $\sim$ aquacentre/aec/publications/; last accessed, 24 Sept 2004).

Nash, L.T., Fritz, J., Alford, P.A. and Brent, L. (1999) Variables influencing the origins of diverse abnormal behaviors in a large sample of captive chimpanzees (Pan troglodytes). American Journal of Primatology 48, 15-29.

Nieuwenhuys, R. (1994) The neocortex. Anatomy and Embryology 190, 307-337.

Nimchinsky, E.A., Vogt, B.A., Morrison, J.H. and Hof, P.R. (1997) Neurofilament and calcium-binding proteins in the human cingulate cortex. The Journal of Comparative Neurology 384, 597-620.

Odling-Smee, L. and Braithwaite, V.A. (2003) The role of learning in fish orientation. Fish and Fisheries 4, 235-246.

Overmier, J.B. and Hollis, K.L. (1990) Fish in the think tank: learning, memory, and integrated behavior. In: Neurobiology of Comparative Cognition (eds R.P. Kesner and D.S. Olson), Lawrence Erlbaum, Hillsdale, NJ, pp. 205-236.

Parvizi, J. and Damasio, A. (2001) Consciousness and the brainstem. Cognition 79, 135-159.

Portavella, M., Vargas, J.P., Torres, B. and Salas, C. (2002) The effects of telencephalic pallial lesions on spatial, temporal, and emotional learning in goldfish. Brain Research Bulletin 57, 397-399.

Price, D.D., Barrell, J.J. and Rainville, P. (2002) Integrating experiential-phenomenological methods and neuroscience to study neural mechanisms of pain and consciousness. Consciousness and Cognition 11, 593-608.

Rolls, E.T. (2000) Précis of the brain and emotion. Behavioral and Brain Sciences 23, 177-234.

Rose, J.D. (2002) The neurobehavioral nature of fishes and the question of awareness and pain. Reviews in Fisheries Science 10, 1-38.

Saper, C.B. (2002) The central autonomic nervous system: conscious visceral perception and autonomic pattern generation. Annual Review of Neuroscience 25, 433-469.

Schiff, N.D., Ribary, U., Moreno, D.R. et al. (2002) Residual cerebral activity and behavioural fragments can remain in the persistently vegetative brain. Brain 125, 1210-1234.

Schilhab, T.S.S. (2002) Anthropomorphism and mental state attribution. Animal Behaviour 63, 1021-1026.

Searle, J.R. (1998) How to study consciousness scientifically. Philosophical Transactions of the Royal Society of London: Series B 353, 1935-1942.

Searle, J.R. (2000) Consciousness. Annual Review of Neuroscience 23, 557-578.
Sewards, T.V. and Sewards, M.A. (2000) Visual awareness due to neuronal activities in subcortical structures: a proposal. Consciousness and Cognition 9, 86-116.

Sewards, T.V. and Sewards, M.A. (2001) On the correlation between synchronized oscillatory activities and consciousness. Consciousness and Cognition 10, 485495.

Shettleworth, S.J. (2001) Animal cognition and animal behaviour. Animal Behaviour 61, 277-286.

Sneddon, L.U. (2003) The evidence for pain in fish: the use of morphine as an analgesic. Applied Animal Behaviour Science 83, 153-162.

Sneddon, L.U., Braithwaite, V.A. and Gentle, M.J. (2003) Do fishes have nociceptors? Evidence for the evolution of a vertebrate sensory system. Proceedings of the Royal Society of London, Series B 270, 1115-1121

Sporns, O., Tononi, G. and Edelman, G. (1991) Modeling perceptual grouping and figures-ground segregation by means of active reentrant connections. Proceedings of the National Academy of Sciences of the USA 88, 129-133.

Striedter, G.F. (2002) Brain homology and function: an uneasy alliance. Brain Research Bulletin 57, 239-242.

Tononi, G. and Edelman, G. (1998) Consciousness and complexity. Science 282, 1846-1851.

Tononi, G., Sporns, O. and Edelman, G. (1994) A measure for brain complexity: relating functional segregation and integration in the nervous system. Proceedings of the National Academy of Sciences of the USA 91, 5033-5037.

Tononi, G., Sporns, O. and Edelman, G.M. (1999) Measures of degeneracy and redundancy in biological networks. Proceedings of the National Academy of Sciences of the USA 96, 3257-3262.

Topál, J. and Csányi, V. (1999) Interactive learning in the paradise fish (Macropodus opercularis): an ethological interpretation of the second-order conditioning paradigm. Animal Cognition 2, 197-206.

Treede, R.-D., Kenshalo, D.R., Gracely, R.H. and Jones, A.K.P. (1999) The cortical representation of pain. Pain 79, 105-111.

Vallortigara, G. (2000) Comparative neuropsychology of the dual brain: a stroll through animals' left and right perceptual worlds. Brain and Language 73, 189-219.

Willis, W.D. and Westlund, K.N. (1997) Neuroanatomy of the pain system and of the pathways that modulate pain. Journal of Clinical Neurophysiology 14, 2-31.

Wray, G.A. (2002) Do convergent developmental mechanisms underlie convergent phenotypes. Brain, Behavior and Evolution 59, 327-336.

Zeman, A. (2001) Consciousness. Brain 124, 1263-1289. Zerbolio, D.J. and Royalty, J.L. (1983) Matching and oddity conditional discrimination in the goldfish as avoidance responses: evidence for conceptual avoidance learning. Animal Learning and Behavior 11, 341-348. 